\section{P3.270 ANTIMICROBIAL SUSCEPTIBILITY AND MOLECULAR CHARACTERIZATION OF NEISSERIA GONORRHOEAE STRAINS FROM SLOVENIA 2008-2012}

doi:10.1136/sextrans-2013-051184.0726

${ }^{1}$ S Jeverica, ${ }^{2} \mathrm{D}$ Golparian, ${ }^{3} \mathrm{M}$ Matičič, ${ }^{4} \mathrm{M}$ Potočnik, ${ }^{5} \mathrm{~B}$ Mlakar, ${ }^{2} \mathrm{M}$ Unemo. ${ }^{1}$ Institute of Microbiology and Immunology, Faculty of Medicine, University of Ljubljana, Ljubljana, Slovenia; ${ }^{2}$ WHO Collaborating Centre for Gonorrhoea and other Sexually Transmitted Infections, Department of Laboratory Medicine, Microbiology, Örebro University Hospital, Örebro, Sweden; ${ }^{3}$ Clinic for Infectious Diseases and Febrile IIInesses, University Medical Centre Ljubljana, Ljubljana, Slovenia., Ljubljana, Slovenia; ${ }^{4}$ Clinic for Dermatovenereology, University Medical Centre Ljubljana, Ljubljana, Slovenia; ${ }^{5}$ Surgical Centre Zdrav Splet, Ljubljana, Slovenia

Objectives Antimicrobial resistance in Neisseria gonorrhoeae is a major public health problem worldwide. Slovenia is among the countries with highest prevalence of decreased susceptibility and resistance to extended-spectrum cephalosporins (ESCs) in Europe. Herein, the phenotypic and molecular characterization of Slovenian N. gonorrhoeae strains from 2008-2012 is presented.

Methods N. gonorrhoeae isolates cultured 2008-2012 in Slovenia $(n=138)$ were examined for antimicrobial susceptibility with Etest methodology for 8 antimicrobials (cefixime, ceftriaxone, penicillin, ciprofloxacin, azithromycin, tetracycline, gentamicin and spectinomycin). Furthermore, all isolates were investigated with Neisseria gonorrhoeae multi-antigen sequence typing (NG-MAST) for molecular epidemiology, and sequencing of major ESC resistance determinants; penA, mtrR and penB.

Results The overall prevalence of resistance and decreased susceptibility to cefixime and ceftriaxone (MIC $\geq 0.125 \mathrm{mg} / \mathrm{L}$ ) was $16 \%$ and $7 \%$, respectively. The resistance and decreased susceptibility showed an epidemic peak during 2009-2011 when it reached 28\% for cefixime and $12 \%$ for ceftriaxone, however, the prevalence subsequently decreased to $6 \%$ and 4\%, respectively, in the year 2012 . NG-MAST sequence types 1407 (13\% of all isolates), 21 (8\%) and 225 (6\%) were the most common STs during 2008-2012. ST1407, previously stated as an internationally spread successful clone with resistance or decreased susceptibility to ESCs, was most prevalent in 2009 (43\%). However, the ST1407 prevalence declined in 2010 $(15 \%)$ and $2011(11 \%)$ and the clone was rare in 2012 (4\%). Instead, in 2012 the ESC susceptible ST21 was the predominant ST (21\%). During 2008-2012, a penA mosaic allele (mainly penA XXXIV, associated with ST1407) was detected in $25 \%$ of isolates.

Conclusion The prevalence of gonococcal resistance to ESC in Slovenia has fluctuated, which is mainly due to the longitudinal prevalence of a few gonococcal clones. Fortunately, some ESC susceptible clones, such as ST21, now appear to replace the main ESC resistant clone ST1407, a replacement that is indicated in several European countries.

\section{P3.271 IDENTICAL MULTILOCUS SEQUENCE TYPING (MLST) ANALYSIS IN SEQUENTIAL SAMPLES FROM PATIENTS WITH PHARYNGEAL CHLAMYDIA INFECTIONS}

doi:10.1136/sextrans-2013-051184.0727

\begin{abstract}
${ }^{1,2,3} \mathrm{M}$ S van Rooijen, ${ }^{3} \mathrm{~N}$ Nassir, ${ }^{3} \mathrm{R}$ Bom, ${ }^{1,4,5} \mathrm{H} \mathrm{J} \mathrm{C}$ de Vries, ${ }^{3} \mathrm{~S}$ Bruisten, ${ }^{3} \mathrm{~A}$ P van Dam. 'STI Outpatient Clinic, Public Health Service of Amsterdam (GGD Amsterdam), Amsterdam, The Netherlands; ${ }^{2}$ Department of Research, Public Health Service of Amsterdam IGGD Amsterdam), Amsterdam, The Netherlands; ${ }^{3}$ Public Health Laboratory, Public Health Service of Amsterdam (GGD Amsterdam), Amsterdam, The Netherlands; ${ }^{4}$ Center for Infection and Immunology Amsterdam (CINIMA), Academic Medical Center (AMC), University of Amsterdam, Amsterdam, The Netherlands; ${ }^{5}$ Department of Dermatology, Academic Medical Center (AMC), University of Amsterdam, Amsterdam, The Netherlands
\end{abstract}

Introduction Pharyngeal Chlamydia trachomatis (PCt) must persist to contribute to ongoing transmission. In a retrospective study, we examined MLST-types of PCt in patients who had a positive pharyngeal swab on two visits, and had not been treated for this infection at first visit.

Methods From 1/1/2008 to 14/7/2010, pharyngeal swabs from patients at risk for pharyngeal gonorrhoea were tested with the AC2 (Hologic-GenProbe) test. Since at that time PCt detection was not considered to represent an infection, PCt results were not reported and patients were not treated, unless they had a chlamydial infection at another anatomic site. We looked for patients who had a positive PCt test on two different occasions with an interval of at least 3 weeks. For inclusion in the study, patients were required to have no Chlamydia infections at other anatomic locations at first visit and therefore received no treatment. PCt typing was done by MLST on stored specimens.

Results Sixteen patients could be included and paired pharyngeal samples from four of those patients contained enough DNA for MLST analysis. The intervals between the two visits were 112,168, 207 and 268 days, respectively. In all four patients MLST types of both pharyngeal samples were completely identical. Patients were two women and two men who had sex with men (MSM). At second visit one woman and one MSM reported commercial sex work and had 30 and 150 sexual partners in the last 6 months, respectively. The second woman reported sex with two known persons and the second MSM reported sex with 15 known persons. None reported sex with a steady partner.

Conclusion Our findings of identical MLST types are consistent with persistent PCt infection for a period of 3-9 months, although repetitive exposure to untreated partners with identical C. trachomatis strains can not be excluded.

\section{P3.272 PREVALENCE OF CHLAMYDIA TRACHOMATIS, NEISSERIA GONORRHOEAE AND UREAPLASMA UREALYTICUM IN PREGNANT WOMEN OF SABZEVAR - IRAN}

doi:10.1136/sextrans-2013-051184.0728

${ }^{1} \mathbf{M}$ Haghighi Hasanabad, ${ }^{2} \mathrm{~A}$ Bahador, ${ }^{1} \mathrm{M}$ Mohammadzadeh, ${ }^{3} \mathrm{~F}$ Haghighi. ${ }^{1} \mathrm{Cellular}$ and Molecular Biology Research Center, Sabzevar University of Medical Sciences, Sabzevar, Iran; 'Department of Microbiology, School of Medicine, Tehran University of Medical Sciences, Tehran, Iran, Tehran, Iran; ${ }^{3}$ Department of Microbiology, Faculty of Public Health, Tehran University of Medical Sciences, Tehran, Iran

Background One approach to eliminating sexually transmitted Infections (STIs) in a community is to screen high-risk persons, followed by the treatment and education of people who test positive. We examined a Triplex PCR assay to detect urine samples in pregnant women. major goal of this study was to determine the prevalence of Chlamydia trachomatis (Ct.), Neisseria gonorrhoeae (Ng.) and Ureaplasma Urealyticum (Uu.) and to apply a Triplex PCR techniques to detect 3 pathogens in one specimens.

Methods A cross-sectional study was conducted among 399 pregnant adolescents women. All participants received prenatal care and delivered at urban hospital in Sabzevar- Iran. A socio- demographic questionnaire was completed. A Triplex PCR with universal primers was developed for screening of subjected women in this study. Descriptive and univariate analyses were performed to describe disease prevalence.

Results Of pregnant adolescents, $15.28 \%$ were diagnosed with either Ct., $\mathrm{Ng}$. or Uu. infection. The prevalence of Ct. was $12.28 \%$, and that of $\mathrm{Ng} 1.25 \%$ and finally $19.54 \%$ of pregnant had $\mathrm{Uu}$. infection.

In univariate analysis, $\mathrm{Ct}$. was associated with having had any level of education $(\mathrm{P}<0.05)$, abortion $(\mathrm{P}<0.05)$, and $\mathrm{Uu}$. was associated with PTD $(\mathrm{P}<0.05)$. Multivariate analysis did not show any significant association.

Conclusions Compared with available data, a decline of STIs prevalence was observed in our setting. This might be the result of 